\title{
AN EXAMPLE WHERE TOPOLOGICAL ENTROPY IS CONTINUOUS
}

BY

\author{
LOUIS BLOCK
}

\begin{abstract}
ABSTRACr. Let ent denote topological entropy, and let $C^{r}\left(S^{1}, S^{1}\right)$ denote the space of continuous functions of the circle to itself having $r$ continuous derivatives with the $C^{r}$ (uniform) topology. Let $f_{0}$ denote a particular $C^{2}$ map of the circle ( $f_{0}$ is the first bifurcation point one comes to in a bifurcation from a full three shift to a map with finite nonwandering set). The main results of this paper are the following:

THEOREM A. The map ent: $C^{0}\left(S^{1}, S^{1}\right) \rightarrow R \cup\{\infty\}$ is lower-semicontinuous at $f_{0}$.

THeOREM B. The map ent: $C^{2}\left(S^{1}, S^{1}\right) \rightarrow R$ is continuous at $f_{0}$.

In proving these two theorems several general results on entropy of mappings of the circle are proved.
\end{abstract}

1. Introduction and statement of results. This paper is concerned with topological entropy of mappings of the circle. After proving some general results we show that for an example of a map $f_{0} \in C^{2}\left(S^{1}, S^{1}\right)$, topological entropy (considered as a map from $C^{2}\left(S^{1}, S^{1}\right)$ to the nonnegative real numbers) is continuous at $f_{0}$. The example is nontrivial in the sense that entropy is not locally constant at $f_{0}$. The main results will be stated precisely after a description of the example is given (see Theorems A,B,C, and D).

Let $f_{0}$ denote any map in $C^{2}\left(S^{1}, S^{1}\right)$ that satisfies the following conditions (see $\$ 2$ for definitions and see Figure 1 for a picture):

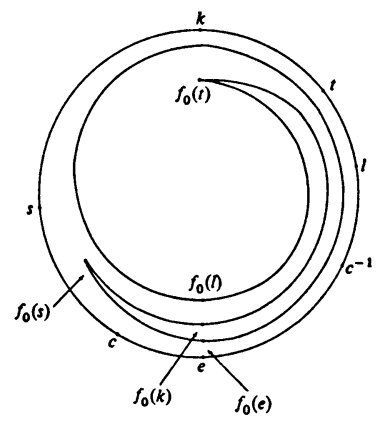

FIGURE 1

Presented to the Society, November 1, 1975; received by the editors February 12, 1976. AMS (MOS) subject classifications (1970). Primary 58F99, 58F15, 54H20.

- American Mathematical Society 1977 
(1) $f_{0}$ has an expanding fixed point $e$, and a contracting fixed point $c$, and $f_{0}$ is orientation preserving at $e$ and $c$.

(2) $f_{0}$ has nondegenerate singularities $t$ and $s$, and these are the only singularities of $f_{0}$.

(3) There are points $l, k \in S^{1}$ with $f_{0}(l)=f_{0}(k)=e$.

(4) The points $e, l, t, k, s, f_{0}(s)$, and $c$ are distinct, and are in order around the circle in the counter-clockwise direction.

(5) $f_{0}$ is one to one on each of the intervals $(e, l),(l, t),(t, s),(s, e)$. Here $(a, b)$ denotes the open arc from $a$ counter-clockwise to $b$.

(6) $(k, e)$ is in the stable manifold of $c$.

(7) $f_{0}(t)=k$.

The map $f_{0}$ is pictured in Figure 1. In Figure 1, we have drawn the image of $f_{0}$ inside $S^{1}$, but of course $f_{0}$ maps $S^{1}$ onto itself.

Let $C^{r}\left(S^{1}, S^{1}\right)$ denote the space of continuous functions of the circle to itself having $r$ continuous derivatives, with the $C^{r}$ (uniform) topology. Let ent denote topological entropy (see $\$ 2$ for definition). Our main results are the following.

TheOREM A. The map ent: $C^{0}\left(S^{1}, S^{1}\right) \rightarrow R \cup\{\infty\}$ is lower-semicontinuous at $f_{0}$.

TheOREM B. The map ent: $C^{2}\left(S^{1}, S^{1}\right) \rightarrow R$ is continuous at $f_{0}$.

In proving Theorems A and B we obtain some general results of independent interest on entropy of mappings of the circle. We discuss some of these in the outline of the paper which follows.

In $\$ 3$ we prove the following theorem:

THeOREM C. Let $f \in C^{1}\left(S^{1}, S^{1}\right)$. Suppose there is a subset $W$ of $S^{1}$, and a finite collection of proper closed intervals of $S^{1},\{I(1), I(2), \ldots, I(p)\}$, which satisfy the following:

(1) Either $W=S^{1}$ or $W=S^{1}-\left(O_{1} \cup \cdots \cup O_{m}\right)$ where $O_{i}$ is a component of the stable manifold of a contracting periodic point $C_{i}$ for $i=1, \ldots, m$.

(2) $W=I(1) \cup \cdots \cup I(p)$.

(3) For $j=1, \ldots, p, f$ maps $I(j)$ homeomorphically onto its image.

(4) For any $i=1, \ldots, p$ and $j=1, \ldots, p, f(I(i)) \cap I(j)$ is an interval.

Then ent $(f) \leqslant \ln (p)$.

Note that given any $C^{1}$ map of the circle with finitely many singularities, from the basic picture of the map (i.e., a picture as in Figure 1) one can obtain (by using Theorem C) an upper bound for the entropy of the map. In particular, Theorem $C$ implies that if $g$ is near $f_{0}$ in $C^{2}\left(S^{1}, S^{1}\right)$ then ent $(g) \leqslant$ $\ln (3)$ (see the proof of Theorem B in $\$ 7$ for details).

Let $f \in C^{1}\left(S^{1}, S^{1}\right)$ and suppose that $f$ has a singularity, and that all 
singularities of $f$ are in the stable manifolds of sinks. In $\$ 4$ we obtain a matrix $A$ associated to $f$ (see Definition 6, the choice of $A$ is not unique) and prove the following theorem.

TheOREM D. ent $(f)=\ln (\lambda(A))$ where $\lambda(A)$ is the largest eigenvalue of $A(\ln$ denotes the natural logarithm).

In $\$ 5$ we construct a sequence $\left(f_{n}\right)$ of maps in $C^{2}\left(S^{1}, S^{1}\right)$, and we show (using Theorem D) that ent $\left(f_{n}\right)$ approaches $\ln (3)$ as $n$ approaches infinity. Each $f_{n}$ has the same basic picture as $f_{0}$, but $f_{n}(t) \in(t, k)$.

In $\$ 6$ we prove a comparison theorem, which we use in $\$ 7$ to show that for $n$ arbitrarily large, if $g$ is close enough to $f_{0}$ in $C^{0}\left(S^{1}, S^{1}\right)$ then ent $(g) \geqslant$ ent $\left(f_{n}\right)$. Combining these results we obtain Theorems $\mathrm{A}$ and $\mathrm{B}$, along with the fact that ent $\left(f_{0}\right)=\ln (3)$. It is easy to see that entropy is not locally constant at $f_{0}$ (see Proposition 9 of $\S 5$ for details).

Topological entropy was defined by Adler, Konheim, and McAndrew in [1]. Other papers on the subject include [2], [3], [4], [6], and [7]. In [2], it is shown that entropy as a map on $C^{0}\left(S^{1}, S^{1}\right)$ is not continuous at any map with finite entropy, while, in [6], an example is given where entropy is not continuous in the diffeomorphism case (on a manifold of dimension three).

Continuity of entropy may be thought of as a kind of quantitative stability in the sense that for dynamical systems near the given one the "amount of action" will be close to the given one.

Note that if one perturbs the map $f_{0}$ to an Axiom A map $g$ (with only one sink $c$ ), whose basic picture is similar to $f_{0}$ except that $g(t) \in(k, c)$, then $\Omega(g)-\{c\}$ will be equivalent to the full one-sided shift on three symbols (see $\$ 2$ for definitions). So $f_{0}$ may be thought of as the first bifurcation point one comes to, in a bifurcation from the full shift on three symbols to a map with finite nonwandering set.

Based on the results of this paper we make the following conjecture.

CONJECTURE E. If $f \in C^{2}\left(S^{1}, S^{1}\right)$ and all singularities of $f$ are nondegenerate then topological entropy (as a map from $C^{2}\left(S^{1}, S^{1}\right.$ ) to the nonnegative real numbers) is continuous at $f$.

We also pose the following questions.

(1) Is topological entropy continuous everywhere on $C^{2}\left(S^{1}, S^{1}\right)$, or even $C^{1}\left(S^{1}, S^{1}\right)$ ?

(2) If $M$ is a compact manifold is the property, topological entropy is continuous at $f$, a generic property of $C^{r}(M, M)$ (for suitable $r$ )?

I would like to thank R. F. Williams for helpful conversations on this subject, and Candy Block for drawing the figures.

2. Preliminary definitions and results. Let $f \in C^{1}\left(S^{1}, S^{1}\right)$. A point $x \in S^{1}$ is called a periodic point of $f$ if $f^{n}(x)=x$ for some positive integer $n$. The 
minimum of $\left\{n: f^{n}(x)=x\right\}$ is called the period of $x$.

A periodic point $x$ of period $n$ is called a sink or contracting periodic point if $\left|D f^{n}(x)\right|<1$. In this case we define the stable manifold of $x$, denoted $W^{s}(x)$, by

$$
W^{s}(x)=\left\{y \in S^{1}: \lim _{k \rightarrow \infty} f^{n k}(y)=x\right\} .
$$

If $y \in W^{s}(x)$ we define the semilocal stable manifold of $y(\operatorname{denoted} \operatorname{slsm}(y))$ to be the component of $W^{s}(x)$ which contains $y$.

A periodic point $x$ of period $n$ is called expanding if $\left|D f^{n}(x)\right|>1$.

A point $x \in S^{1}$ is called a singularity of $f$ if $D f(x)=0$. A singularity $x$ is said to be nondegenerate if $D^{2} f(x) \neq 0$.

Now, we review the definition of topological entropy given in [1]. Let $X$ be a compact topological space and $f: X \rightarrow X$ a continuous map. For any two open covers $\mathbb{Q}$ and $\mathscr{B}$ of $X$ let $\mathscr{Q} \vee \mathscr{B}$ denote $\{A \cap B: A \in \mathbb{Q}$ and $B \in \mathscr{B}\}$, and let $f^{-1}(\mathscr{Q})$ denote $\left\{f^{-1}(A): A \in \mathbb{Q}\right\}$. Let $M_{n}(f, \mathbb{Q})$ denote the minimum cardinality of a subcover of $X$ of $\mathscr{Q} \vee f^{-1}(\mathscr{Q}) \vee \cdots \vee f^{(-n+1)}(\mathbb{Q})$. We set

$$
\operatorname{ent}(f, \mathbb{Q})=\lim _{n \rightarrow \infty} \frac{1}{n}\left(\ln \left(M_{n}(f, \mathbb{Q})\right)\right)
$$

where ln denotes the natural logarithm. It is easy to see that this limit exists and is finite (see [1]). Finally we define the topological entropy of $f$ by

$$
\operatorname{ent}(f)=\sup (\operatorname{ent}(f, \mathbb{Q}))
$$

where the supremum is taken over all open covers $\mathbb{Q}$ of $X$. If $X$ is a metric space it suffices to consider any sequence of open covers whose diameter approaches zero (see [1]). By the diameter of an open cover $\mathbb{Q}$ we mean the supremum of the diameters of the open sets in $Q$.

Two of the basic properties of topological entropy are the following (see [1]).

Proposition 1. Let $X$ and $Y$ be compact topological spaces, and let $f: X \rightarrow X$ and $g: Y \rightarrow Y$ be continuous maps. Suppose there is a continuous onto map $h$ : $Y \rightarrow X$ such that $f(h(y))=h(g(y))$ for all $y \in Y$. Then $\operatorname{ent}(f) \leqslant \operatorname{ent}(g)$. If $h$ is a homeomorphism (in which case $f$ and $g$ are said to be topologically conjugate) then $\operatorname{ent}(f)=\operatorname{ent}(g)$.

Proposition 2. If $f: X \rightarrow X$ is a continuous map of a compact topological space $X$, and $K$ is a closed subset of $X$ with $f(K) \subset K$, then $\operatorname{ent}(f) \geqslant \operatorname{ent}(f \mid K)$.

Let $f: X \rightarrow X$ be continuous. A point $x \in X$ is said to be wandering if there is a neighborhood $V$ of $x$ with $f^{n}(V) \cap V=\varnothing \forall n>0$. The set of points which are not wandering is called the nonwandering set and denoted $\Omega(f)$. We state the following proposition from [4]. 
Proposition 3. Let $f: X \rightarrow X$ be a continuous map of a compact metric space. Then $\operatorname{ent}(f)=\operatorname{ent}(f \mid \Omega(f))$.

We next review some terminology concerning subshifts of finite type. Let $A$ be an $n$ by $n$ matrix with all entries zero or one. Let $X_{A}$ denote the set of infinite sequences $\left(i_{0} i_{1} i_{2} \ldots\right)$ such that for each nonnegative integer $j$, $i_{j} \in\{1,2, \ldots, n\}$ and $A\left(i_{j}, i_{j+1}\right)=1$, where $A\left(i_{j}, i_{j+1}\right)$ denotes the entry of $A$ in row $i_{j}$ and column $i_{j+1}$. We have a map $s_{A}: X_{A} \rightarrow X_{A}$ given by

$$
s_{A}\left(. i_{0} i_{1} i_{2} \ldots\right)=\left(. i_{1} i_{2} i_{3} \ldots\right) \text {. }
$$

This map is called the one-sided subshift of finite type associated to $A$. (If $A$ is the $n$ by $n$ matrix with all entries one, $s_{A}$ is called the full shift on $n$ symbols.)

Note that $X_{A}$ is a compact topological space and $s_{A}: X_{A} \rightarrow X_{A}$ is continuous. The topology on $X_{A}$ is induced by the metric

$$
d\left(\left(. i_{0} i_{1} i_{2} \ldots\right),\left(. j_{0} j_{1} j_{2} \ldots\right)\right)=\sum_{k=0}^{\infty} 2^{-k}\left(\delta\left(i_{k} j_{k}\right)\right)
$$

where $\delta\left(i_{k}, j_{k}\right)=1$ if $i_{k} \neq j_{k}$ and $\delta\left(i_{k} j_{k}\right)=0$ if $i_{k}=j_{k}$.

We conclude this section by stating the following well-known proposition (see [4]).

Proposition 4. For any subshift of finite type $s_{A}: X_{A} \rightarrow X_{A}$, ent $\left(s_{A}\right)=\ln (\lambda)$, where $\lambda$ is the largest eigenvalue of $A$.

\section{Proof of Theorem $\mathbf{C}$.}

LEMMA 5. Let $f \in C^{0}\left(S^{1}, S^{1}\right)$. Let $K_{1}, \ldots, K_{n}$ be proper closed intervals of $S^{1}$, such that for each $i=1, \ldots, n-1, f \mid K_{i}$ is a homeomorphism and $f\left(K_{i}\right) \subset$ $K_{i+1}$. Let $\mathbb{Q}$ be a covering of $K_{1} \cup \cdots \cup K_{n}$ by finitely many open intervals, such that $\forall A \in \mathbb{Q}$ and $i=1, \ldots, n, A \cap K_{i}$ is either an interval or empty. Let $\operatorname{card}(\mathbb{Q})=k$ (card denotes cardinality). Then there is a subset $\mathscr{B}$ of $\mathbb{Q} \vee$ $f^{-1}(\mathbb{Q}) \vee \cdots \vee f^{(-n+1)}(\mathbb{Q})$ which covers $K_{1}$, with $\operatorname{card}(\mathscr{B}) \leqslant n \cdot k$.

Proof. For each $i=1, \ldots, n$ choose a subset $\mathcal{Q}_{i}$ of $\mathbb{Q}$ which is a cover of $K_{i}$ of minimum cardinality. Let $Q_{i}=\left\{A_{i}(1), A_{i}(2), \ldots, A_{i}\left(r_{i}\right)\right\}$ for $i=$ $1, \ldots, n$. Let $K_{i}(j)=A_{i}(j) \cap K_{i}$ for $i=1, \ldots, n$ and $j=1, \ldots, r_{i}$, and let $\mathrm{C}_{i}=\left\{K_{i}(1), K_{i}(2), \ldots, K_{i}\left(r_{i}\right)\right\}$ for $i=1, \ldots, n$.

Let $K_{i}=\left[a_{i}, b_{i}\right]$ for $i=1, \ldots, n$. For $x$ and $y$ in $K_{i}$ let $d_{i}(x, y)$ denote the length of the arc joining $x$ and $y$ which is in $K_{i}$. If $Y$ is a subset of $K_{i}$ and $x \in K_{i}$, let $d_{i}(x, Y)$ denote $\inf \left\{d_{i}(x, y): y \in Y\right\}$.

By renumbering the elements of each $\mathcal{C}_{i}$ (and each $Q_{i}$ ) we may assume that the following are true (using the minimality of $Q_{i}$ ):

(1) $0=d_{1}\left(a_{1}, K_{1}(1)\right)<d_{1}\left(a_{1}, K_{1}(2)\right)<\cdots<d_{1}\left(a_{1}, K_{1}\left(r_{1}\right)\right)$,

(2) for $p=2, \ldots, n$, 


$$
0=d_{p}\left(a_{p}, K_{p}(1)\right)<d_{p}\left(a_{p}, K_{p}(2)\right)<\cdots<d_{p}\left(a_{p}, K_{p}\left(r_{p}\right)\right)
$$

if $f^{(p-1)}$ is orientation preserving on $K_{1}$ while

$$
0=d_{p}\left(b_{p}, K_{p}(1)\right)<d_{p}\left(b_{p}, K_{p}(2)\right)<\cdots<d_{p}\left(b_{p}, K_{p}\left(r_{p}\right)\right)
$$

if $f^{(p-1)}$ is orientation reversing on $K_{1}$.

Let $B_{i}(j)=K_{i}(j)-\left(K_{i}(1) \cup \cdots \cup K_{i}(j-1)\right)$ for $i=1, \ldots, n$ and $j=$ $1, \ldots, r_{i}$. Then $B_{i}(1) \cup \cdots \cup B_{i}\left(r_{i}\right)=K_{i}$ and $B_{i}(j) \cap B_{i}(m)=\varnothing$ for $j \neq$ $m$. Also it follows from (1) and (2) above that the following is true:

(3) Suppose $p<m, j_{1} \leqslant j_{3}, f^{(m-p)}\left(B_{p}\left(j_{1}\right)\right) \cap B_{m}\left(j_{2}\right) \neq \varnothing$, and $f^{(m-p)}\left(B_{p}\left(j_{3}\right)\right) \cap B_{m}\left(j_{4}\right) \neq \varnothing$. Then $j_{2} \leqslant j_{4}$.

Let $\left(j_{1} j_{2}, \ldots, j_{n}\right)$ denote $B_{1}\left(j_{1}\right) \cap f^{-1}\left(B_{2}\left(j_{2}\right)\right) \cap \cdots \cap f^{(-n+1)}\left(B_{n}\left(j_{n}\right)\right)$ where $1 \leqslant j_{i} \leqslant r_{i}$ for $i=1, \ldots, n$. We write $\left(j_{1} j_{2}, \ldots, j_{n}\right)<\left(i_{1}, i_{2}, \ldots, i_{n}\right)$ if $j_{m}<i_{m} \forall m=1, \ldots, n$ while for some $k=1, \ldots, n, j_{k}<i_{k}$.

It follows from (3) above that if $\left(j_{1} j_{2}, \ldots, j_{n}\right)$ and $\left(i_{1}, i_{2}, \ldots, i_{n}\right)$ are nonempty and distinct then either $\left(j_{1} j_{2}, \ldots, j_{n}\right)<\left(i_{1}, i_{2}, \ldots, i_{n}\right)$ or $\left(i_{1}, i_{2}, \ldots, i_{n}\right)<\left(j_{1} j_{2}, \ldots, j_{n}\right)$. Hence we may list the nonempty distinct sets of the form $\left(j_{1} j_{2}, \ldots, j_{n}\right)$ in order from the smallest to the largest (using the order $<$ ). It follows from the definition of $<$ that we can have at most $r_{1}+r_{2}+\cdots+r_{n}$ sets on the list. Since $r_{i} \leqslant k$ for $i=1, \ldots, n$, we have $r_{1}+r_{2}+\cdots+r_{n} \leqslant n \cdot k$. Thus there are at most $n \cdot k$ nonempty distinct sets of the form $\left(j_{1}, j_{2}, \ldots, j_{n}\right)=B_{1}\left(j_{1}\right) \cap f^{-1}\left(B_{2}\left(j_{2}\right)\right) \cap \cdots \cap$ $f^{(-n+1)}\left(B_{n}\left(j_{n}\right)\right)$.

Let $\mathscr{B}$ denote the collection of sets of the form $A_{1}\left(j_{1}\right) \cap f^{-1}\left(A_{2}\left(j_{2}\right)\right)$ $\cap \cdots \cap f^{(-n+1)}\left(A_{n}\left(j_{n}\right)\right)$ such that $\left(j_{1} j_{2}, \ldots, j_{n}\right)$ is nonempty. Then $\mathscr{B}$ is a subset of $\mathscr{Q} \vee f^{-1}(\mathscr{Q}) \vee \cdots \vee f^{(-n+1)}(\mathscr{Q})$ which covers $K_{1}$ and $\operatorname{card}(\mathscr{B})<$ $n \cdot k$. Q.E.D.

THEOREM C. Let $f \in C^{1}\left(S^{1}, S^{1}\right)$. Suppose there is a subset $W$ of $S^{1}$, and a finite collection of proper closed intervals of $S^{1},\{I(1), I(2), \ldots, I(p)\}$, which satisfy the following:

(1) Either $W=S^{1}$ or $W=S^{1}-\left(O_{1} \cup O_{2} \cup \cdots \cup O_{m}\right)$ where $O_{i}$ is a component of the stable manifold of a contracting periodic point $c_{i}$ for $i=$ $1, \ldots, m$.

(2) $W=I(1) \cup I(2) \cup \cdots \cup I(p)$.

(3) For $j=1, \ldots, p, f$ maps $I(j)$ homeomorphically onto its image.

(4) For any $i=1, \ldots, p$ and $j=1, \ldots, p, f(I(i)) \cap I(j)$ is an interval.

Then $\operatorname{ent}(f) \leqslant \ln (p)$.

Proof. Let

$$
\delta=\min \left\{l\left(S^{1}-I(1)\right), \ldots, l\left(S^{1}-I(p)\right)\right\}
$$

where $l\left(S^{1}-I(j)\right)$ denotes the length of the interval $S^{1}-I(j)$. Let $\mathbb{Q}$ be 
any finite cover of $I(1) \cup I(2) \cup \cdots \cup I(p)$ by open intervals with the diameter of $\mathbb{Q}$ less than $\delta$. Let $k=\operatorname{card}(\mathbb{Q})$.

Let $I\left(j_{1} j_{2}, \ldots, j_{n}\right)=I\left(j_{1}\right) \cap f^{-1}\left(I\left(j_{2}\right)\right) \cap \cdots \cap f^{(-n+1)}\left(I\left(j_{n}\right)\right)$ where $j_{i}$ $\in\{1,2, \ldots, p\} \forall i=1, \ldots, n$. Then each nonempty $I\left(j_{1} j_{2}, \ldots, j_{n}\right)$ is a closed interval and $f$ maps $I\left(j_{1} j_{2}, \ldots, j_{n}\right)$ homeomorphically into $I\left(j_{2}, \ldots, j_{n}\right)$. For any fixed $I\left(j_{1} j_{2}, \ldots, j_{n}\right)$, by Lemma 5 (with $K_{i}=$ $\left.I\left(j_{i}, \ldots, j_{n}\right)\right)$, there is a subset of $\mathscr{Q} \vee f^{-1}(\mathbb{Q}) \vee \cdots \vee f^{(-n+1)}(\mathbb{Q})$ which covers $I\left(j_{1}, j_{2}, \ldots, j_{n}\right)$ of cardinality at most $n \cdot k$.

Let $X=S^{1}-\left(\cup_{i=1}^{m} W^{s}\left(c_{i}\right)\right)$. Then $X$ is a compact set with $f(X) \subset X$ and $f^{-1}(X) \subset X$.

Let $\mathscr{Q}(X)$ be the open cover of $X$ defined by $\mathscr{Q}(X)=\{A \cap X: A \in \mathbb{Q}\}$. The minimal number of open sets of $\mathscr{Q}(X) \vee f^{-1}(\mathscr{Q}(X)) \vee \cdots \vee$ $f^{(-n+1)}(\mathscr{Q}(X))$ needed to cover $X$ is equal to the minimal number of open sets of $\mathscr{Q} \vee f^{-1}(\mathbb{Q}) \vee \cdots \vee f^{(-n+1)}(\mathbb{Q})$ needed to cover $X$. Now $X$ is contained in the union of all the $I\left(j_{1} j_{2}, \ldots, j_{n}\right)$. Also, there are at most $p^{n}$ distinct intervals of the form $I\left(j_{1}, j_{2}, \ldots, j_{n}\right)$ and each one of these may be covered by a subset of $\mathbb{Q} \vee f^{-1}(\mathbb{Q}) \bigvee \cdots \vee f^{(-n+1)}(\mathbb{Q})$ of cardinality at most $n \cdot k$. Hence $M_{n}(f \mid X, \mathbb{Q}(X)) \leqslant p^{n} \cdot n \cdot k$. This implies that ent $(f \mid X, \mathbb{Q}(X)) \leqslant \ln (p)$.

Since the diameter of $\mathscr{Q}(X)$ may be taken to be arbitrarily small, we have $\operatorname{ent}(f \mid X) \leqslant \ln (p)$. But $X$ contains all nonwandering points of $f$ except for the finite set $\left\{c_{1}, c_{2}, \ldots, c_{m}\right\}$. Thus

$$
\operatorname{ent}(f)=\operatorname{ent}(f \mid \Omega(f))=\operatorname{ent}(f \mid X) \leqslant \ln (p) \text {. Q.E.D. }
$$

\section{Proof of Theorem $D$.}

Definition 6. Let $f \in C^{1}\left(S^{1}, S^{1}\right)$ and suppose that $f$ has a sink and all singularities of $f$ are in the stable manifolds of sinks. Let $A$ be an $m$ by $m$ matrix. We say $A$ is a matrix associated to $f$ if there is a collection $\left\{I_{1}, \ldots, I_{m}\right\}$ of pairwise disjoint closed intervals (or points) on $S^{1}$ such that the following hold:

(1) For each $j=1, \ldots, m, f\left(I_{j}\right) \neq S^{1}$.

(2) Let $W=S^{1}-\left(I_{1} \cup \cdots \cup I_{m}\right)$. Then each component of $W$ is a component of the stable manifold of a sink.

(3) $f(W) \subset W$.

(4) $W$ contains all singularities of $f$.

(5) $A(i, j)=1$ if $f\left(I_{i}\right) \cap I_{j} \neq \varnothing$ and $A(i, j)=0$ otherwise. Here $A(i, j)$ denotes the entry of the matrix $A$ in row $i$ and column $j$.

Note that conditions (3) and (4) of the definition imply that if $f\left(I_{i}\right) \cap I_{j} \neq$ $\varnothing$ then $f\left(I_{i}\right) \supset I_{j}$. Also (1) and (4) imply that $f \mid I_{j}$ is a homeomorphism for $j=1, \ldots, m$.

If $f \in C^{1}\left(S^{1}, S^{1}\right)$ and $f$ has a sink, and all singularities of $f$ are in the stable manifolds of sinks (and if $S^{1}$ is not the stable manifold of a sink), then there 
is a matrix associated to $f$. To obtain a matrix $A$ associated to $f$ note that the set of singularities of $f$, being compact, must be contained in finitely many components of stable manifolds of sinks. Let $W$ be the union of all components of stable manifolds of sinks which contain points in the orbit of a singularity. Then $W$ consists of finitely many components of stable manifolds of sinks and conditions (2), (3), and (4) (of Definition 6) are satisfied. We may add finitely many components of stable manifolds of sinks to $W$ (if necessary) so that if $I_{1}, \ldots, I_{m}$ are the components of $S^{1}-W$, then condition (1) is satisfied along with conditions (2), (3), and (4). Finally we use condition (5) to define the matrix $A$.

In the next section we will see some examples of mappings of the circle with associated matrices.

We remark that if $f$ is expanding on the intervals $I_{1}, \ldots, I_{m}$ and if $K(f)=\left\{x \in S^{1}: f^{n}(x) \in I_{1} \cup \cdots \cup I_{m} \forall n>0\right\}$ then $f \mid K(f)$ is topologically conjugate to the one-sided subshift of finite type $s_{A}$ (see [5]). It is known (see [4]) that $\operatorname{ent}\left(s_{A}\right)=\ln (\lambda(A))$ where $\lambda(A)$ is the largest eigenvalue of $A$. Thus in this case (using Propositions 2 and 3) it follows that ent $(f)=$ $\ln (\lambda(A))$. In the more general case where $f$ may not be expanding on the intervals $I_{1}, \ldots, I_{m}, f \mid K(f)$ need not be conjugate to the shift $s_{A}$. In Theorem $\mathrm{D}$ we show that it is still true that $\operatorname{ent}(f)=\ln (\lambda(A))$.

We will use the following elementary linear algebra fact in the proof of Theorem D.

Proposition 7. Let $A$ be an $m$ by $m$ matrix with nonnegative entries. Let $\lambda(A)$ be the largest eigenvalue of $A$ and let $S_{n}$ denote the sum of the entries of $A^{n}$. Then

$$
\lim _{n \rightarrow \infty}\left(S_{n}\right)^{(1 / n)}=\lambda(A) .
$$

Proof. Define a norm on the space of all $m$ by $m$ matrices by setting $\|B\|=\Sigma|B(i, j)|$ where the sum is taken over all entries of $B$. It is easy to see that this satisfies the properties of a norm. Let $s(A)$ denote the spectral radius of $A$.

Since the matrix $A$ is nonnegative, $\left\|A^{n}\right\|=S_{n}$ and $s(A)=\lambda(A)$. The conclusion now follows from the well-known theorem of analysis

$$
\lim _{n \rightarrow \infty}\left\|A^{n}\right\|^{(1 / n)}=s(A) \text {. Q.E.D. }
$$

THEOREM D. Let $f \in C^{1}\left(S^{1}, S^{1}\right)$. Suppose that $f$ has a sink and all singularities of $f$ are in the stable manifolds of sinks. Let $A$ be a matrix associated to $f$ in the sense of Definition 6. Then $\operatorname{ent}(f)=\ln (\lambda(A))$, where $\lambda(A)$ is the largest eigenvalue of $A$.

Proof. Let $I_{1}, \ldots, I_{m}$ be the closed intervals which determine the matrix $A$ 
in the sense of Definition 6. Let $K(f)=\left\{x \in S^{1}: f^{n}(x) \in\left(I_{1} \cup \cdots \cup I_{m}\right)\right.$ $\forall n>0\}$. Then $\operatorname{ent}(f)=\operatorname{ent}(f \mid K(f))$ since $K(f)$ contains all but finitely many points of $\Omega(f)$.

Let $\mathscr{B}$ be the open cover of $K(f)$ given by $\mathscr{B}=\left\{I_{1} \cap K(f), \ldots, I_{m} \cap\right.$ $K(f)\}$. We will show

$$
\operatorname{ent}(f \mid K(f))=\operatorname{ent}(f \mid K(f), \Re) \text {. }
$$

Note that $M_{n}(f \mid K(f), \mathscr{B})$ is equal to the number of nonempty distinct sets of the form $I_{j_{1}} \cap f^{-1}\left(I_{j_{2}}\right) \cap \cdots \cap f^{(-n+1)}\left(I_{j_{n}}\right)$. This is true because any two distinct sets of this form are disjoint, and any nonempty set of this form must contain an element of $K(f)$.

Let $\mathscr{Q}$ be any open cover of $K(f)$ with the property that for each $A \in \mathbb{Q}$ there is an open interval $V$ such that $A=V \cap K(f)$ and $V \cap I_{j}$ is an interval for $j=1, \ldots, m$. Let $\operatorname{card}(\mathbb{Q})=k$. By using Lemma 5 as in the proof of Theorem $\mathrm{C}$ we obtain

$$
M_{n}(f \mid K(f), \mathscr{Q}) \leqslant n \cdot k \cdot M_{n}(f \mid K(f), \mathscr{B}) .
$$

Hence ent $(f \mid K(f), \mathbb{Q}) \leqslant \operatorname{ent}(f \mid K(f), \mathscr{B})$. Since the diameter of $\mathscr{Q}$ may be arbitrarily small we have ent $(f \mid K(f))=\operatorname{ent}(f \mid K(f), \mathscr{B})$.

It follows from condition (5) of Definition 6 that $M_{n}(f \mid K(f), \mathscr{B})$ is the sum of the entries of the matrix $A^{n}$. Thus by Proposition 7, ent $(f \mid K(f), \mathscr{B})=$ $\ln (\lambda(A))$. We have

$$
\operatorname{ent}(f)=\operatorname{ent}(f \mid K(f))=\operatorname{ent}(f \mid K(f), \mathscr{B})=\ln (\lambda(A)) \text {. Q.E.D. }
$$

5. Construction of the sequence $\left(f_{n}\right)$. In this section we construct a sequence $\left(f_{n}\right)$ of maps in $C^{2}\left(S^{1}, S^{1}\right)$ with ent $\left(f_{n}\right) \rightarrow \ln (3)$ as $n \rightarrow \infty$.

Let $f_{n}$ be any map in $C^{2}\left(S^{1}, S^{1}\right)$ which satisfies conditions (1)-(6) of the definition of $f_{0}$ in $\$ 1$ and also the following condition. Let $c^{0}=c$. For $i=1,2, \ldots, n-1$, let $c^{-i}$ denote the unique inverse image (under $f_{n}$ ) of $c^{(-i+1)}$ in $(e, l)$, and let $c^{-n}$ denote the unique inverse image (under $f_{n}$ ) of $c^{-(n-1)}$ in $(t, k)$. Then we require $f_{n}$ to satisfy the condition that $f_{n}(t) \in$ $\operatorname{slsm}\left(c^{-n}\right)$. (Recall slsm $\left(c^{-n}\right)$ denotes the component of $W^{s}(c)$ which contains $c^{-n}$.)

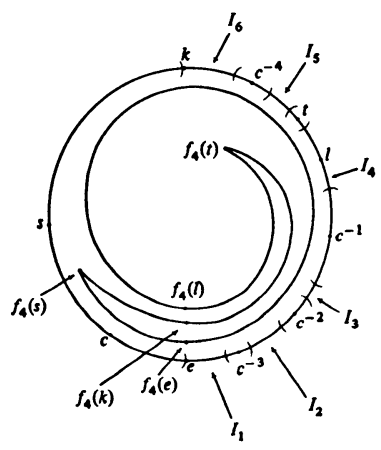

FIGURE 2 
The map $f_{4}$ is pictured in Figure 2. In Figure 2, the open intervals indicated about $c^{-3}, c^{-2}, c^{-1}, t$, and $c^{-4}$ represent the semilocal stable manifolds of these points.

Let $I_{1}, I_{2}, I_{3}, I_{4}, I_{5}$, and $I_{6}$ be the components of the complement in $S^{1}$ of $\operatorname{slsm}(c) \cup \operatorname{slsm}\left(c^{-1}\right) \cup \operatorname{slsm}\left(c^{-2}\right) \cup \operatorname{slsm}\left(c^{-3}\right) \cup \operatorname{slsm}\left(c^{-4}\right) \cup \operatorname{slsm}(t)$ where we order these intervals as in Figure 2. Using these closed intervals we obtain a matrix $A_{4}$ associated to $f_{4}$ (see Definition 6 of $\S 4$ ) and

$$
A_{4}=\left(\begin{array}{llllll}
1 & 1 & 0 & 0 & 0 & 0 \\
0 & 0 & 1 & 0 & 0 & 0 \\
0 & 0 & 0 & 1 & 1 & 1 \\
1 & 1 & 1 & 1 & 1 & 0 \\
0 & 1 & 1 & 1 & 1 & 0 \\
1 & 0 & 0 & 0 & 0 & 0
\end{array}\right) .
$$

For $n \geqslant 4$, we may obtain a matrix $A_{n}$ associated to $f_{n}$ in the same manner as we did with $f_{4}$, and $A_{n}$ is the $n+2$ by $n+2$ matrix

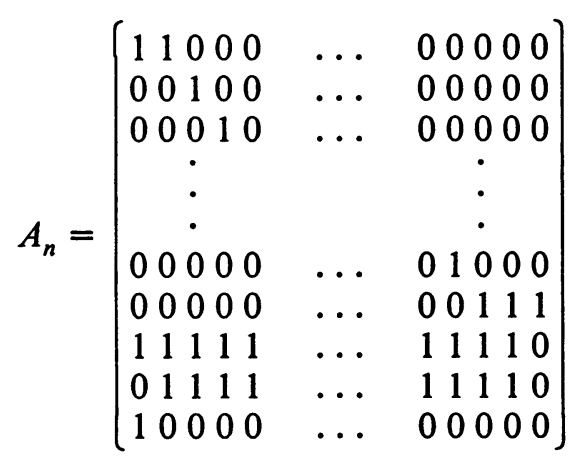

where the missing rows have ones on the super-diagonal and zeros elsewhere.

A straightforward calculation shows that

$$
\operatorname{det}\left(A_{n}-\lambda I\right)=( \pm 1)\left(\lambda^{n+2}-3 \lambda^{n+1}+2 \lambda\right),
$$

where $I$ denotes the $n+2$ by $n+2$ identity matrix. Let $a_{n}$ denote the largest root of this polynomial (the largest eigenvalue of $\left.A_{n}\right)$. Then $\left(a_{n}\right)^{n+1}-3\left(a_{n}\right)^{n}$ $+2=0$. This implies $\left(a_{n}\right)^{n}\left(3-a_{n}\right)=2$. Since as $n \rightarrow \infty,\left(a_{n}\right)^{n} \rightarrow \infty$ it follows that as $n \rightarrow \infty, a_{n} \rightarrow 3$. But $\operatorname{ent}\left(f_{n}\right)=\ln \left(a_{n}\right)$ by Theorem $\mathrm{D}$, so that $\operatorname{ent}\left(f_{n}\right) \rightarrow \ln (3)$ as $n \rightarrow \infty$. This is what we wanted to prove.

We state this result formally in the following.

Proposition 8. For the sequence $\left(f_{n}\right)$ constructed above ent $\left(f_{n}\right) \rightarrow \ln (3)$ as $n \rightarrow \infty$.

We next use the maps $f_{n}$ to verify that (as noted in §1) entropy is not locally constant at $f_{0}$. 
Proposition 9. The map ent: $C^{2}\left(S^{1}, S^{1}\right) \rightarrow R$ is not locally constant at $f_{0}$.

Proof. It is easy to see that arbitrarily close to $f_{0}\left(\right.$ in $\left.C^{2}\left(S^{1}, S^{1}\right)\right)$ there is a map $g_{N}$ which satisfies the same conditions as some map $f_{N}$ in the sequence defined above. Thus ent $\left(g_{N}\right)=\ln \left(a_{N}\right)$ where $a_{N}$ is the largest eigenvalue of $A_{N}$. From the proof of Proposition 8, we have $\left(a_{N}\right)^{N}\left(3-a_{N}\right)=2$, so $a_{N}<3$. Hence ent $\left(g_{N}\right)<\ln (3)$. We will show in the proof of Theorem A (in $\$ 7$ ) that $\operatorname{ent}\left(f_{0}\right)=\ln (3)$ so this proves Proposition 9. Q.E.D.

\section{A comparison theorem.}

THEOREM 10. Let $f \in C^{1}\left(S^{1}, S^{1}\right)$. Suppose $f$ has a sink, and all singularities of $f$ are in the stable manifolds of sinks (and suppose $S^{1}$ is not the stable manifold of a sink). Let $A$ be a matrix associated to $f$ and let $I_{1}, \ldots, I_{m}$ be the disjoint closed intervals which define the matrix $A$ (see Definition 6). Let $g \in C^{0}\left(S^{1}, S^{1}\right)$ and suppose there are disjoint closed intervals $J_{1}, \ldots, J_{m}$ such that $f\left(I_{j}\right) \supset I_{k}$ implies that $g\left(J_{j}\right) \supset J_{k}$. Then $\operatorname{ent}(g) \geqslant \operatorname{ent}(f)$.

Proof. Let $s_{A}: X_{A} \rightarrow X_{A}$ denote the one-sided subshift of finite type associated to $A$ (see $\$ 2$ for definition). Let $K(g)$ denote the set of $x \in S^{1}$ that satisfy the following two conditions:

(1) $g^{k}(x) \in J_{1} \cup \cdots \cup J_{m}$ for each nonnegative integer $k$.

(2) If $g^{k}(x) \in J_{i_{k}}$ for each nonnegative integer $k$ then $\left(. i_{0} i_{1} i_{2} \ldots\right) \in X_{A}$.

Note that $K(g)$ is a closed subset of $S^{1}$. Define $h: K(g) \rightarrow X_{A}$ by $h(x)=\left(. i_{0} i_{1} i_{2} \ldots\right)$ where $g^{k}(x) \in J_{i_{k}}$ for each nonnegative integer $k$.

We show that $h$ is onto. Suppose $\left(. i_{0} i_{1} i_{2} \ldots\right) \in X_{A}$. Then $f\left(I_{i_{k}}\right) \supset I_{i_{k+1}}$ for $k=0,1,2, \ldots$. Hence $g\left(J_{i_{k}}\right) \supset J_{i_{k+1}}$ for $k=0,1,2, \ldots$ It follows that there is at least one point $x \in S^{1}$ with $g^{k}(x) \in J_{i_{k}}$ for $k=0,1,2, \ldots$ (using the fact that a decreasing intersection of nonempty compact sets is nonempty). By definition $h(x)=\left(. i_{0} i_{1} i_{2} \ldots\right)$ so $h$ is onto.

$h$ is continuous because for any positive integer $N$ and $x \in K(g)$, if $y \in K(g)$ is sufficiently close to $x$, then $g^{k}(x)$ and $g^{k}(y)$ will be in the same $J_{i}$ for $0 \leqslant k \leqslant N$, so $h(x)$ and $h(y)$ will agree on the first $N$ places. Clearly $s_{A} \circ h=h \circ g$, so by Propositions 1,2, and 4 and Theorem D,

$$
\operatorname{ent}(g) \geqslant \operatorname{ent}(g \mid K(g)) \geqslant \operatorname{ent}\left(s_{A}\right)=\operatorname{ent}(f) \text {. Q.E.D. }
$$

\section{Proof of Theorems $A$ and $B$.}

TheOREM A. The map ent: $C^{0}\left(S^{1}, S^{1}\right) \rightarrow R \cup\{\infty\}$ is lower-semicontinuous at $f_{0}$.

Proof. Let $\varepsilon>0$. Pick $N$ so large that $\ln (3)-\operatorname{ent}\left(f_{N}\right)<\varepsilon$ (where $f_{N}$ is the $N$ th term of the sequence constructed in $\S 5)$. Pick $\delta>0$ such that if $d\left(f_{0}, g\right)<\delta$ (where $d$ is a metric on $C^{0}\left(S^{1}, S^{1}\right)$ ) then the following seven conditions are satisfied. 
(1) There are points $e(g), c(g), l(g)$, and $k(g)$ on $S^{1}$ corresponding to the points $e, c, l$, and $k$ for $f_{0}$, i.e. $e(g)$ is a fixed point of $g$ near $e, c(g)$ is a fixed point of $g$ near $c$, and $l(g)$ and $k(g)$ are points near $l$ and $k$ respectively with $g(l(g))=g(k(g))=e(g)$.

(2) $g$ maps the interval $[e(g), l(g)]$ onto $S^{1}$.

(3) There is a point $t(g) \in(l(g), k(g))$ such that for any $x \in[l(g), k(g)]$, $g(t(g)) \in[g(x), c(g))$.

(4) There is a point $c^{-1}(g)$ in $(e(g), l(g))$ with $g\left(c^{-1}(g)\right)=c(g)$ and with the property that the image under $g$ of the interval $\left(e(g), c^{-1}(g)\right)$ contains the interval $(e(g), c(g))$, but is not equal to $S^{1}$.

(5) $g(t(g)) \in\left(c^{-N}(g), c(g)\right)$ where $c^{-N}(g)$ is any point of $S^{1}$ satisfying the following. For $i=2, \ldots, N-1$ let $c^{-i}(g)$ be a point in $\left(e(g), c^{(-i+1)}(g)\right)$ with $g\left(c^{-i}(g)\right)=c^{(-i+1)}(g)$. Then let $c^{-N}(g)$ be a point in $(t(g), k(g))$ with $g\left(c^{-N}(g)\right)=c^{(-N+1)}(g)$.

(6) The image under $g$ of the interval $\left(c^{-1}(g), t(g)\right)$ contains the interval $\left(c(g), c^{-N}(g)\right)$ but is not equal to $S^{1}$.

(7) The image under $g$ of the interval $(t(g), k(g))$ contains the interval $\left(e(g), c^{-N}(g)\right)$ but is not equal to $S^{1}$.

These conditions imply that the basic picture of $g$ will be the same as that of $f_{0}$ in Figure 1, except that $g(t(g))$ need not be $k(g)$, and $g$ may have many new "turning points".

Let $I_{1}, I_{2}, \ldots, I_{N+2}$ be the disjoint closed intervals which are the components of the complement in $S^{1}$ of $\operatorname{slsm}(c) \cup \operatorname{slsm}\left(c^{-1}\right) \cup \cdots \cup \operatorname{slsm}\left(c^{-N}\right)$ $U \operatorname{slsm}(t)$ where everything is defined for $f_{N}$ as in $\$ 5$, i.e. $c^{0}=c$ and for $i=1, \ldots, N-1, c^{-i}$ is the unique point in $(e, l)$ with $f_{N}\left(c^{-i}\right)=c^{(-i+1)}$, and $c^{-N}$ is the unique point in $(t, k)$ with $f_{N}\left(c^{-N}\right)=c^{(-N+1)}$. Also we take $I_{1}$ to be the closed interval which contains $e, I_{2}$ the next one in the counter-clockwise direction, etc. (see Figure 2 for the case $N=4$ ).

Let $g \in C^{0}\left(S^{1}, S^{1}\right)$ with $d\left(f_{0}, g\right)<\delta$. Let $O(c(g))$ denote the open interval $(k(g), e(g))$. Let $O\left(c^{0}(g)\right)=O(c(g))$. For $i=1, \ldots, N$ let $O\left(c^{-i}(g)\right)$ denote the component of $f^{-1}\left(O\left(c^{(-i+1)}(g)\right)\right)$ which contains $c^{-i}(g)$. Let $O(t(g))$ denote any open interval about $t(g)$ such that $g(O(t(g))) \subset$ $\left(c^{-N}(g), c(g)\right)$.

Let $J_{1} J_{2}, \ldots, J_{N+2}$ be the disjoint closed intervals which are the components of the complement in $S^{1}$ of $O(c(g)) \cup O\left(c^{-1}(g)\right) \cup \cdots \cup$ $O\left(c^{-N}(g)\right) \cup O(t(g))$ where the $J_{i}$ are ordered in the same manner as the $I_{i}$. Then $f_{N}\left(I_{j}\right) \supset I_{k}$ implies that $g\left(J_{j}\right) \supset J_{k}$ (see Figure 2 of $\S 5$ for the case $N=4)$, so ent $(g) \geqslant \operatorname{ent}\left(f_{N}\right)$ by Theorem 10 of $\$ 6$.

By the same argument as above it follows that ent $\left(f_{0}\right) \geqslant \operatorname{ent}\left(f_{n}\right)$ for any integer $n>2$. Hence by Proposition 8, ent $\left(f_{0}\right) \geqslant \ln (3)$.

By Theorem $C$ of $\$ 3$, ent $\left(f_{0}\right) \leqslant \operatorname{Ln}(3)$. To apply Theorem $C$ we take 
$I(1)=\left[e, c^{-1}\right], I(2)=\left[c^{-1}, t\right]$, and $I(3)=[t, k]$ (see Figure 1). Thus ent $\left(f_{0}\right)$ $=\ln (3)$.

We have

$$
\operatorname{ent}\left(f_{0}\right)-\operatorname{ent}(g)=\ln (3)-\operatorname{ent}(g) \leqslant \ln (3)-\operatorname{ent}\left(f_{N}\right)<\varepsilon \text {. Q.E.D. }
$$

TheOREM B. The map ent: $C^{2}\left(S^{1}, S^{1}\right) \rightarrow R$ is continuous at $f_{0}$.

Proof. Let $d_{2}$ denote a metric on $C^{2}\left(S^{1}, S^{1}\right)$. Let $\varepsilon>0$. By Theorem A, $\exists \delta_{1}>0$ such that if $g \in C^{2}\left(S^{1}, S^{1}\right)$ with $d_{2}\left(g, f_{0}\right)<\delta_{1}$, then ent $\left(f_{0}\right)-\operatorname{ent}(g)$ $<\varepsilon$. By Theorem C, $\exists \delta_{2}>0$ such that if $g \in C^{2}\left(S^{1}, S^{1}\right)$ with $d_{2}\left(g, f_{0}\right)<\delta_{2}$ then ent $(g) \leqslant \ln (3)$. Here we are using the fact that for $g$ sufficiently close to $f_{0}$ in $C^{2}\left(S^{1}, S^{1}\right), g$ will have exactly two singularities corresponding to those of $f_{0}$, and we apply Theorem $\mathrm{C}$ as in the proof of Theorem A. Also, by the proof of Theorem A, ent $\left(f_{0}\right)=\ln (3)$.

Let $\delta=\min \left\{\delta_{1}, \delta_{2}\right\}$. Then if $d_{2}\left(g, f_{0}\right)<\delta$, we have $\left|\operatorname{ent}(g)-\operatorname{ent}\left(f_{0}\right)\right|=$ $\operatorname{ent}\left(f_{0}\right)-\operatorname{ent}(g)<\varepsilon$. Q.E.D.

Finally, we remark that the entropy of a continuous map of the circle may be infinite (see $\$ 5$ of [2] for an example), but the entropy of a $C^{1}$ map of the circle is finite (see Proposition 12 of [3]).

\section{REFERENCES}

1. R. L. Adler, A. Konheim and M. McAndrew, Topological entropy, Trans. Amer. Math. Soc. 114 (1965), 309-319. MR 30 \#5291.

2. L. Block, Noncontinuity of topological entropy of maps of the Cantor set and of the interval, Proc. Amer. Math. Soc. 50 (1975), 388-393. MR 51 \# 4195.

3. Rufus Bowen, Entropy for group endomorphisms and homogeneous spaces, Trans. Amer. Math. Soc. 153 (1971), 401-414. MR 43 \#469.

4. Robert Bowen, Topological entropy and Axiom A, Proc. Sympos. Pure Math., vol. 14, Amer. Math. Soc., Providence, R. I., 1970, pp. 23-41. MR 41 \#7066.

5. M. V. Jakobson, On smooth mappings of the circle into itself, Mat. Sb. 85(127) (1971), 163-188 = Math. USSR Sbornik 14 (1971), 161-185. MR 44 \#7587.

6. M. Misiurewicz, On non-continuity of topological entropy, Bull. Acad. Polon. Sci. Sér. Sci. Math. Astronom. Phys. 19 (1971), 319-320. MR 44 \# 4781.

7. M. Shub and R. F. Williams, Entropy and stability (preprint).

8. S. Smale, Differentiable dynamical systems, Bull. Amer. Math. Soc. 73 (1967), 747-817. MR 37 \# 3598; erratum, 39, p. 1593.

Department of Mathematics, University of Florida, Gainesville, Florida 32611 